\title{
Las redes sociales en las marcas turísticas de la Comunidad Valenciana ${ }^{1}$
}

\author{
Mar IGLESIAS GARCÍA \\ Universidad de Alicante \\ mar.iglesias@ua.es \\ María Dolores FERNÁNDEZ POYATOS \\ Universidad de Alicante \\ dolores.fernandez@ua.es
}

Recibido: 30/07/2012

Aceptado: 23/10/2012

\section{Resumen}

El turismo es uno de los sectores más relevantes de la economía de la Comunidad Valenciana; sin embargo, desde 2009, se produce un importante descenso en la llegada de turistas que motivan la puesta en marcha de diversos planes estratégicos y de competitividad, con propuestas de comunicación en las que resulta fundamental la adaptación a las nuevas tecnologías. En este trabajo se analiza cómo están comunicando, y en qué redes sociales, las principales marcas turísticas de la Comunidad Valenciana: Benidorm, Castellón Mediterráneo, Costa Blanca y València Terra i Mar. Los resultados indican que estas redes se están utilizando más como un canal habitual de promoción y difusión de los productos turísticos, que para el objetivo que las ha hecho populares: la participación e interactividad con el público.

Palabras clave: Redes sociales, marcas turísticas, Comunidad Valenciana.

\section{Social networks in tourist brands in the Valencian Community}

\begin{abstract}
Tourism is one of the most important economical sectors of the Valencian Community, but since 2009, there is a significant drop in tourists arrivals which motivates the implementation of several strategic and competitiveness plans, with new proposals of communication in which it is essential the adaptation to new technologies. This paper analyzes how they are communicating, what social networks are being used, leading tourist brands of Valencian Community: Benidorm, Castellón Mediterráneo, Costa Blanca and Valencia Terra i Mar. The results indicate that these networks are being used more as a normal channel of promotion and dissemination of tourist products, than for the purpose that made them popular: participation and interaction with the public.
\end{abstract}

Keywords: Social networks, tourism brands, Valencian Community

\section{Referencia normalizada}

IGLESIAS GARCÍA, Mar y FERNÁNDEZ POYATOS, María Dolores (2012): "Las redes sociales en las marcas turísticas de la Comunidad Valenciana”. Estudios sobre el mensaje periodístico. Vol. 18, núm. especial noviembre, págs.: 533-541. Madrid, Servicio de Publicaciones de la Universidad Complutense.

Sumario: 1. Introducción; 1.1. La dinámica turística en la Comunidad Valenciana; 1.2. Los planes turísticos de la Conselleria de Turisme de la Comunidad Valenciana; 1.3. Las nuevas tecnologías y las redes sociales en turismo. 2. Metodología. 3. Desarrollo. 4. Conclusiones. 5. Referencias bibliográficas

${ }^{1}$ Este trabajo forma parte del proyecto I+D GV/2012/006 de la Conselleria de Educación de la Generalitat Valenciana - Estrategias y eficacia de la comunicación en el turismo de la Comunidad Valenciana - , dirigido por la Dra. Fernández Poyatos. 


\section{Introducción}

\subsection{La dinámica turística en la Comunidad Valenciana. 2005-2011}

En España, el año 2008 supuso una ruptura del ciclo expansivo del turismo internacional iniciado en el año 2005; a la cifra de 59.193.000 turistas, alcanzada en 2007, le siguió una caída de casi dos millones al año siguiente y de cuatro millones y medio en el 2010, lo que significó una disminución de once puntos porcentuales desde 2005 (Frontur, 2005-2010).

La pérdida de turistas internacionales fue también un hecho en la Comunidad Valenciana, cuyas cifras fueron alarmantes en el año 2009 con seiscientos mil turistas menos que el año anterior y bajando de nuevo en 2010 unos setecientos mil.

El mercado británico fue el que más se resintió con cuatro puntos porcentuales menos; los demás mercados emisores se mantuvieron estos tres años estables e incluso el ítem Resto de Europa creció (Monfort, 2009); no obstante, los valores del año 2010 apenas llegaron a los del 2005, siendo lo más significativo el decrecimiento de los mercados británico y alemán. Sin embargo, en el año 2011 se produjo un cambio de coyuntura con una recuperación de turistas internacionales cifrada en 5.383.340, es decir, un incremento del $7,2 \%$ respecto al año anterior.

En lo que respecta al turismo nacional hacia la Comunidad Valenciana, la serie 2005-2011 arroja, igualmente, un balance negativo, con un descenso de 2.560.667 de turistas. Ahora bien, hay que señalar que las mayores caídas no se produjeron en 2009 -como sucedió con los turistas internacionales-, sino a partir del 2007, cuando se registraron 1.490.488 menos que en 2006 y en el 2010 con un descenso de 2.346 .398 turistas respecto al año 2009 .

Estas cifras motivaron la puesta en marcha diversos planes estratégicos, de competitividad y de marketing para la mejora del sector turístico, como el Plan de Competitividad del Sector Turístico de la Comunidad Valenciana 2009-2011. Desde ese entonces, y hasta la actualidad, la Conselleria ha elaborado e implementado diferentes planes de actuación para la mejora del sector turístico.

\subsection{Los planes turísticos de la Conselleria de Turisme de la Comunidad Valenciana}

El proyecto de la moderna distribución en zonas turísticas de la Comunidad Valenciana proviene de dos documentos de planificación: el Libro Blanco del Turismo en la Comunidad Valenciana (1990) y el Plan Director de los Espacios turísticos de la Comunidad Valenciana (1995). Aunque este último se presentó como documento base para impulsar un nuevo modelo de desarrollo turístico "equilibrado y basado en la gestión racional de los recursos" (Vera et al, 1995: 439), nunca llegó a desarrollarse. Fue necesario esperar al Plan de Espacios turísticos de la Comunidad Valenciana (2008) para iniciar una planificación turística de la Comunidad a fin de mejorar la competitividad del sector.

Las directrices del Plan comprenden medidas que afectan a diversas áreas de actuación; de los ochos ejes planteados, en el séptimo - Promoción, información y comercialización - , queda patente el impulso a Internet y a las nuevas tecnologías de la comunicación (Conselleria de Turisme, 2008: 86), donde destaca la potenciación del uso del portal "comunitatvalenciana.com" para la promoción y comercialización. 
Desde entonces, la Generalitat Valenciana ha puesto llevado a cabo diversos planes estratégicos y operativos. Las razones más inmediatas que lo justifican son la competencia de mercados emergentes y el descenso de turistas desde el año 2008.

El Plan de competitividad del sector turístico de la Comunidad Valenciana 20092011 se articuló en torno a siete ejes, siendo el quinto el de «Promoción, comunicación y posicionamiento en mercado». La puesta en marcha de las acciones de dicho Plan se materializó en dos documentos operativos: el Plan de imagen y posicionamiento marketing 2009 y el Plan de marketing turístico 2010.

En el primero, se reconocía la necesidad de aunar estrategias de creación de producto con las de comunicación y comercialización de los mismos, como acciones primordiales para alcanzar éxitos en el corto y medio plazo. El objetivo general fue "Posicionar la Comunitat Valenciana como un destino de tendencia, cuyo principal punto fuerte es el producto de sol y playa diferencial y rodeado de valor añadido, en el que se suceden grandes eventos, en constante evolución, diversificado y sostenible, donde confluyen en equilibrio la vanguardia y la tradición" (Conselleria de Turisme, 2009: 24).

Como canales de comunicación se consideraron Internet, campañas de publicidad, patrocinios, ferias, workshops, publicaciones de material de promoción y presentaciones de destino. Se contemplaron, pues, tanto acciones tradicionales en el turismo como la publicidad y las relaciones públicas, así como las últimas tendencias comunicativas online.

A pesar de que la campaña de publicidad en medios convencionales fue la acción principal, destacaron por su novedad las actuaciones sobre las nuevas tecnologías: nuevo portal turístico de la Comunidad Valenciana (www.comunitatvalenciana.com), que incorpora un canal audiovisual 2.0, comunidades online (buceo, senderismo, excursionismo), geoposicionamiento (acceso a recursos turísticos a través de Google maps), microsites de municipios, etc.

La continuación lógica del Plan de marketing turístico 2009 fue el Plan de marketing turístico 2010, que siguió la clasificación de los mercados y ejes establecidos con anterioridad y reforzó las inversiones en proyectos de nuevas tecnologías y marketing online; de él, destaca, sobre todo, el impulso otorgado a la estrategia social media a través de la figura del community manager para gestionar y seguir el Social Media Plan del portal de la Comunidad Valenciana.

En el año 2010, se elaboró el Plan estratégico global del turismo de la Comunidad Valenciana 2010-2020, que define la política turística para los próximos diez años y es el documento base del Plan director del turismo de interior 2011-2015 y de los planes operativos y de marketing anuales de la Conselleria de Turisme.

En cuanto al objetivo general, se mantiene el de años precedentes; también los objetivos estratégicos, aunque con una notable diferencia en el enfoque del quinto, ya que por primera vez se basa la promoción "en la segmentación por productos, dirigida a públicos objetivos utilizando formatos tradicionales y en especial las nuevas tecnologías" (Conselleria de Turisme, 2011: 24). En planes anteriores, la promoción se basaba en la segmentación de mercados sin referencia alguna a los medios publicitarios ni a las nuevas tecnologías. 
En cuanto a la comunicación, continúa la campaña global iniciada en el año 2011, cuyo eslogan se enmarca en la estrategia experiencial "Las experiencias intensas hay que compartirlas". El valor experiencial, mencionado en todos los planes analizados, se desarrolla y actualiza en la componente textual de las piezas publicitarias, pero también en otras áreas comunicativas: creación por parte de los usuarios de contenidos experienciales vehiculados a través de redes sociales (Youtube, Facebook...) y comunidades de viajeros (Minube, Tripadvisor).

Si bien el concepto experiencial toma el significado de generar experiencias positivas sobre los destinos de la Comunidad Valenciana y difundirlas en el entorno online a fin de provocar una reacción favorable en los usuarios -potenciales turistas-, hay que recordar que para alcanzar un turismo experiencial se debe mantener la renovación constante de los productos turísticos a un nivel de calidad muy elevado: "Existe un nuevo tipo de turista que se caracteriza, ante todo, por la individualización de gustos y expectativas, pasando de una lógica estandarizada a un consumo experiencial" (Turespaña, 2007: 24). Como no podía ser de otra forma, la apuesta de la comunicación online se está dinamizando e impulsando desde la Conselleria.

\subsection{Las nuevas tecnologías y las redes sociales en turismo}

El desarrollo de las tecnologías de la información, y en los últimos años de las redes sociales, ha transformado la forma de comunicarse y éstas se han convertido en un elemento clave en el que las opiniones de los otros usuarios resultan de fundamental importancia a la hora de tomar decisiones de compra de servicios turísticos. Además, en el proceso de decisión de los usuarios/clientes influye de manera determinante la imagen online que comunican los destinos turísticos. Los internautas intercambian información relativa a sus viajes y de esta manera influyen sobre las opiniones de otros internautas, que participan en una conversación online y que, en el futuro, podrán actuar basándose en las sugerencias, no sólo de amigos o conocidos, sino de una multitud de usuarios/turistas que diariamente cuelgan fotos, videos y comentarios en Internet (Di Placido, 2010: 2). Los usuarios adoptan una nueva posición en el proceso comunicativo, y pasan del rol de mero destinatario de la información a sujeto que actúa en la creación del flujo informativo, crea contenidos y los intercambia con otros cibernautas.

Es por ello por lo que el sector turístico, consciente de la importancia que han adquirido las redes sociales, ha ido creando sus propios perfiles en estas redes, y también las instituciones públicas han elaborado planes de marketing con apartados específicos para impulsarlas y así mejorar la competitividad del sector.

En este estudio se analiza cómo están comunicando, y en qué redes sociales, las principales marcas turísticas de la Comunidad Valenciana, después de la puesta en marcha de diversos planes estratégicos de turismo.

\section{Metodología}

El objeto de análisis que se aborda en este trabajo son las páginas web de las principales marcas turísticas de la Comunidad Valenciana: Benidorm, Castellón Mediterrá- 
neo, Costa Blanca y València Terra i Mar, ${ }^{2}$ y las redes sociales que utilizan. Al estudiar estas webs se ha aplicado el método de investigación descriptivo, basado en el análisis de contenido y la observación estructurada del objeto de estudio en un periodo de tiempo definido. La metodología es cuantitativa, pero los resultados son tanto cuantitativos como cualitativos.

Así, se han analizado los perfiles de estas marcas en las redes sociales más utilizadas (Facebook, Twitter y YouTube) con el fin de extrapolar datos para entender si las administraciones están usando adecuadamente estos espacios online y si están consiguiendo fomentar la actividad informativa de los internautas/turistas.

Para ello se han creado dos fichas de análisis, una para las páginas web y otra para los perfiles en redes sociales. En la ficha de análisis de las páginas web se investiga qué redes sociales tienen implementadas. En la ficha de análisis de los perfiles en redes sociales se analizan los datos relativos a la actividad de los usuarios que se han suscrito a esas redes y también la actividad de los administradores de esos perfiles.

El período de observación y cumplimentación de las fichas, tanto de las webs como de los perfiles en las redes sociales, fue de una semana (del 11 al 17 de junio de 2012). Se presentan a continuación los principales resultados de la información recopilada.

\section{Desarrollo}

Las webs de las marcas turísticas analizadas (Benidorm, Castellón Mediterráneo, Costa Blanca y València Terra i Mar) cuentan con perfiles en las redes sociales Facebook, Twitter y YouTube, que son las que tienen una mayor popularidad. ${ }^{3}$ Además, tres de las webs utilizan Flickr para poner sus álbumes fotográficos (Benidorm, Castellón y Valencia) y los blogs están presentes en Costa Blanca y València Terra y mar (ver tabla 1).

\begin{tabular}{|c|c|c|c|c|c|c|c|c|}
\hline Marca & 5 & 6 & You & 3 & E & (i) & $\bullet$ & Otras \\
\hline Benidorm & Sí & Sí & Sí & No & No & No & Sí & No \\
\hline $\begin{array}{l}\text { Castellón } \\
\text { Mediterráneo / } \\
\text { Turismo de } \\
\text { Castellón }\end{array}$ & Sí & Sí & Sí & Sí & No & Sí & Sí & No \\
\hline Costa Blanca & Sí & Sí & Sí & No & Sí & No & No & $\begin{array}{c}\text { Mi } \\
\text { Nube }\end{array}$ \\
\hline $\begin{array}{l}\text { València Terra } \\
\text { imar }\end{array}$ & Sí & Sí & Sí & No & Sí & No & Sí & No \\
\hline
\end{tabular}

Tabla 1: Redes sociales que tienen las webs de las marcas turísticas valencianas (Mayo 2012). Elaboración propia.

2 http://www.benidorm.es, http://www.turismodecastellon.com, http://www.costablanca.org y http://www.valenciaterraimar.org

${ }^{3}$ Facebook contaba con más de 900 millones de usuarios activos en abril de 2012, 800 millones YouTube y Twitter más de 500 millones de usuarios, en febrero de 2012. 
El uso de otras redes y de herramientas 2.0 es escaso y destaca en este aspecto la web de Castellón Mediterráneo, que también tiene perfiles en Google+ y Tuenti, mientras que Costa Blanca cuenta con un perfil en la comunidad de viajeros MiNube.

Los iconos de las redes sociales, más o menos grandes según la marca, aparecen bien visibles en la página de inicio de todas las webs, y en el caso de Castellón Mediterráneo se destacan los perfiles de Facebook y Twitter con un módulo especial en el menú principal.

En el análisis detallado de los perfiles de Facebook se observa que todos ellos tienen formato abierto (fan page) $)^{4}$ que los internautas pueden agregarse fácilmente pulsando el botón "Me gusta" (like). La fecha de creación de los perfiles coincide aproximadamente en las marcas Benidorm, Castellón Mediterráneo y Costa Blanca, que se iniciaron en junio-julio de 2010, mientras que el perfil más reciente es el de València Terra i mar, que se creó en febrero de 2011 (ver tabla 2).

A pesar de ser el más "joven", el perfil de València Terra i mar es el que, a fecha de junio de 2012, tiene más "amigos", con 6.655 usuarios, seguido por Castellón Mediterráneo (3.683), Costa blanca (3.655) y Benidorm (2.410). No son cifras muy elevadas, si se tiene en cuenta que destinos turísticos valencianos menos conocidos como Altea o Cullera tienen más de dos mil seguidores.

\begin{tabular}{|l|c|c|c|c|}
\hline \multicolumn{1}{|c|}{ Marca } & \multicolumn{1}{c|}{ Perfil en Facebook (FB) } & $\begin{array}{c}\text { Fecha } \\
\text { inicio }\end{array}$ & $\begin{array}{c}\text { N seguidores } \\
\text { mayo }\end{array}$ & Idioma \\
\hline Benidorm & http:/www facebook.com/turismobenidorm & $19 / 07 / 2010$ & 2410 & Esp. \\
\hline $\begin{array}{l}\text { Castellón } \\
\text { Mediterráneo } \\
\text { Turismo de } \\
\text { Castellón }\end{array}$ & http//www facebook.com/turismodecastellon & $25 / 06 / 2010$ & 3683 & Esp. \\
\hline Costa Blanca & http//www facebook.com/costablancaorg & $15 / 06 / 2010$ & 3655 & Esp./Ingl. \\
\hline $\begin{array}{l}\text { València Terra i } \\
\text { mar }\end{array}$ & http//www facebook.com/valenciaterraimar & $12 / 02 / 2011$ & 6655 & Esp. \\
\hline
\end{tabular}

Tabla 2: Características de los perfiles en Facebook de las marcas turísticas valencianas (Junio 2012). Elaboración propia.

El contenido de los mensajes en cada uno de los perfiles es muy similar, con la promoción de actividades culturales y festivas, y en la gran mayoría se ofrecen enlaces, fotografías y vídeos. En cuanto al tipo de lenguaje utilizado por los gestores de los perfiles, coincide en ser directo e informal, en primera persona, animando a los usuarios a que participen en las acciones propuestas. El idioma utilizado es el castellano, aunque en Costa Blanca y Benidorm también usan el inglés en ocasiones. Llama especialmente la atención que apenas hay comentarios, aunque sí hay participación en el botón "Me gusta" de los mensajes.

Por otro lado, en el análisis de los perfiles en Twitter se observa que la marca con más seguidores es Benidorm, con 2.492 agregados. A continuación está Castellón Mediterráneo, con 2.230 , seguido de Costa Blanca (1.320) y por último, València Terra $\mathrm{i}$

${ }^{4}$ Facebook ofrece tres tipos de cuentas: el perfil personal (que es lo que la mayoría de las personas usa) las páginas (fan pages) y los grupos. 
mar, que tiene sólo 369 seguidores (ver tabla 3). El perfil más activo, con 2.212 twitts (mensajes de 140 caracteres) es el de Castellón Mediterráneo, que es además el único que clasifica en listas los otros perfiles que está siguiendo (16 listas). La frecuencia de actualización habitual es 3/4 twitts cada uno dos días.

\begin{tabular}{|c|c|c|c|c|c|c|c|}
\hline Marca & $\begin{array}{c}\text { Perfil Twitter } \\
E\end{array}$ & $\begin{array}{l}\text { Fecha } \\
\text { inicio }\end{array}$ & $\begin{array}{c}\mathrm{N}^{\mathrm{D}} \\
\text { seguidores }\end{array}$ & $\begin{array}{c}\mathrm{N}^{\mathrm{p}} \\
\text { siguiendo }\end{array}$ & $\begin{array}{l}\mathrm{N}^{\mathrm{D}} \text { de } \\
\text { Twitts }\end{array}$ & $\begin{array}{c}N^{0} \\
\text { listas }\end{array}$ & Idioma \\
\hline Benidorm & $\begin{array}{l}\text { (d)turismobenidor } \\
\mathrm{m}\end{array}$ & $10 / 06 / 2010$ & 2492 & 703 & 1224 & 0 & Esp. \\
\hline $\begin{array}{c}\text { Castellón } \\
\text { Mediterráneo / } \\
\text { Turismo de } \\
\text { Castellón }\end{array}$ & (Q)turcastellon & $26 / 01 / 2011$ & 2230 & 1336 & 2212 & 16 & Esp. \\
\hline Costa Blanca & (d)costablancaorg & $17 / 06 / 2010$ & 1320 & 303 & 1598 & 0 & $\begin{array}{c}\text { Esp. Ing } \\
\text { acciones en } \\
\text { el extrajero }\end{array}$ \\
\hline $\begin{array}{l}\text { València Terra } \\
\text { i mar }\end{array}$ & (d)valterraimar & $23 / 05 / 2011$ & 369 & 204 & 570 & 0 & Esp. \\
\hline
\end{tabular}

Tabla 3: Características de los perfiles en Twitter de las marcas turísticas valencianas (Junio 2012). Elaboración propia.

Los perfiles en Twitter de las cuatro marcas están ligados a los perfiles de Facebook y reproducen exactamente los mismos textos y las mismas imágenes en las dos redes sociales, por lo que el idioma en el que se escriben es también mayoritariamente el castellano. Sólo en el perfil de Castellón Mediterráneo se observan algunos retwitts (RT) a mensajes de perfiles de poblaciones de su entorno. Así, se observa que la interacción con los seguidores y con otras marcas es casi inexistente.

También es significativo el número de perfiles a los que sigue cada marca. De nuevo, Castellón Mediterráneo es quien sigue la conversación de más perfiles, con 1336, mientras que Benidorm sigue 703, Costa Blanca 303, y València Terra i mar 204 (ver tabla 3).

Por otro lado, en los canales de YouTube, la marca con más vídeos es Benidorm, con 1.693 piezas (ver tabla 4). Eso se debe principalmente a que usa el canal audiovisual para difundir noticias elaboradas por Benidorm Televisión (BTv). En consecuencia, el número de visualizaciones es también el mayor, con 119.558. Las otras marcas tienen un número mucho menor de vídeos (34 Castellón, con 23.535 visualizaciones; 37 Costa Blanca, con 20.999 visualizaciones y 24 València, con 3.282 visualizaciones). En estos casos, las piezas audiovisuales son sobre todo vídeos promocionales, que se han usado en campañas de televisión. El número de seguidores de los canales es muy bajo en todos los casos. Cabe destacar que el canal de YouTube está enlazado desde los perfiles de Facebook en el caso de Benidorm, Castellón y València Terra i mar.

El idioma que se utiliza en las piezas audiovisuales también es mayoritariamente el castellano, a excepción de Costa Blanca y Benidorm que incluyen también vídeos en inglés y ruso. 


\section{Conclusiones}

Los resultados del análisis indican que se está haciendo un esfuerzo desde las administraciones para implementar las redes sociales en sus webs. Sin embargo, estas redes se están utilizando más como un canal de promoción y difusión de los productos turísticos, que para el objetivo que las ha hecho populares: la participación e interactividad con el público. Es el caso de los perfiles creados en Facebook, desde los que se envían mensajes diariamente, con un estilo directo e informal, pero en los que no se establece una relación de feed-back con los usuarios. Esa situación se reproduce en los perfiles de Twitter, ya que se limitan a difundir exactamente los mismos textos e imágenes que en Facebook, sin promover el diálogo o el intercambio de opiniones. Esto entraría en contradicción con el objetivo, plasmado en los diferentes planes de competitividad y de marketing, de dinamizar la participación y generar contenido por parte de los usuarios.

Llama la atención que no se han incorporado redes como Linkdin, posiblemente porque tiene una imagen de red profesional más que de ocio y entretenimiento, ni tampoco se ha implementado Pinterest, la última red social que está aumentando sus usuarios de manera vertiginosa y que tiene aplicaciones muy interesantes en el sector turístico.

Por otro lado, destaca que, siendo el turismo extranjero un porcentaje importantísimo del público objetivo de estas marcas, la inmensa mayoría de los mensajes emitidos a través de las redes sociales se hace en castellano.

La marca que mayor conocimiento muestra respecto a las posibilidades de las redes sociales es Castellón Mediterráneo, que ha aprovechado la clasificación por listas y hace retwitts, ha implementado Instagram y Pinterest en su perfil de Facebook, ha incorporado un perfil en Google $+\mathrm{y}$, a pesar de tener los perfiles de más reciente creación, ha mejorado las cifras de las otras marcas turísticas en cuanto a número de seguidores (Facebook) o número de twitts, lo que denota un mayor conocimiento de la red, y/o una mayor dedicación, por parte del gestor de los contenidos, aunque también reproduce el error de repetir los mismos mensajes en Facebook y Twitter.

A pesar del claro incremento de la inversión y de las actuaciones por las herramientas 2.0, tal y como recogen los planes estratégicos y de marketing de la Conselleria de Turisme, del estudio realizado se puede ratificar una consideración relativa a la actividad de las administraciones valencianas en materia de promoción turística online: se encuentra todavía en una fase poco madura en lo concerniente a la adopción de instrumentos de la Web 2.0 internamente a sus propias webs, se observa escasa atención o infravaloración hacía una herramienta tan importante como las redes sociales, al fin de conseguir una buena promoción turística.

\section{Referencias bibliográficas}

BERNÉ MANERO, Carmen; GARCÍA GONZÁLEZ, Margarita; GARCÍA UCEDA, M. Esperanza \& MÚGICA GRIJALBA, José Miguel (2011): "La influencia de las TIC en la estructura del sistema de distribución turístico". Cuadernos de Turismo, $\mathrm{n}^{\circ} .28$, pp. 9-22. 
DI PLACIDO, Angelo (2010): Interactividad usuario-usuario y redes sociales online en el sector turístico. Análisis de las páginas web turísticas oficiales de las administraciones andaluzas, en VIII Congreso "Turismo y Tecnologías de la Información y las Comunicaciones" Turitec.

EXCELTUR, CONSELLERIA DE TURISMO: Estudio del impacto económico del turismo sobre la economía y el empleo de la Comunidad Valenciana. Años 2008, 2009 y 2010.

GENERALITAT VALENCIANA (2008): Plan de competitividad del sector turístico de la Comunidad Valenciana 2009-2011. Documento ejecutivo, (julio 2008).

GENERALITAT VALENCIANA, CONSELLERIA DE TURISMO (2009): Plan de imagen y posicionamiento marketing 2009.

GENERALITAT VALENCIANA, CONSELLERIA DE TURISMO (2011): Plan de marketing turístico 2011.

GENERALITAT VALENCIANA, CONSELLERIA DE TURISMO (2012): Plan de marketing turístico 2012.

INSTITUTO DE ESTUDIOS TURÍSTICOS (IET): Informes anuales de Frontur. Años 2005-2010.

MONFORT, Vicente (2009): "Comunidad Valenciana", en AECIT: La actividad turística española en 2010. Madrid, Centro de Estudios R. Areces.

VERA, Fernando; CRUZ, Jorge \& BAÑOS, Carlos Javier (1995): "Turismo y organización del territorio: desajustes de un modelo de implantación y nuevas estrategias". Cuadernos de Geografía, no 58, pp. 439-474.

\section{Mar IGLESIAS GARCÍA}

mar.iglesias@ua.es

Profesora Dra. del Departamento de Comunicación y Psicología Social

Universidad de Alicante

Campus de San Vicente del Raspeig

Edf. CC. Sociales. D64.

Ap. 00 E-03080 Alicante

\section{MaDolores FERNÁNDEZ POYATOS}

dolores.fernandez@ua.es

Profesora Dra. del Departamento de Comunicación y Psicología Social

Universidad de Alicante

Campus de San Vicente del Raspeig

Edf. CC. Sociales. P2046

Ap. 00 E-03080 Alicante 